\title{
Clinicopathological characteristics of neuroendocrine differentiated invasive breast cancer with simultaneous gastric, colon and lung metastases: a case report
}

\author{
Yang-kun Wang ( $\nabla$ yk333wang@126.com ) \\ Foresea Life Insurance Guangzhou General Hospital \\ Xiao-dong Chen \\ Foresea Life Insurance Guangzhou General Hospital \\ Xing-hai Liao \\ Shenzhen Hospital \\ Bin-Feng Yang \\ Xinxiang Central Hospital
}

\section{Case Report}

Keywords: Invasive ductal carcinoma of the breast, Neuroendocrine differentiation, Metastasis, Clinicopathological characteristics, Immunohistochemistry

Posted Date: February 21st, 2022

DOI: https://doi.org/10.21203/rs.3.rs-1374802/v1

License: (c) (1) This work is licensed under a Creative Commons Attribution 4.0 International License.

Read Full License 


\section{Abstract}

Background: To investigate the clinicopathological characteristics of infiltrating ductal carcinoma of the breast with neuroendocrine differentiation and metastasis to the stomach, colon and lung at the same time.

Methods: To The histopathological characteristics and immunophenotype of a case of breast invasive ductal carcinoma with neuroendocrine characteristics and concurrent gastric, colon and lung metastases were observed.

Results: The histological structure and cell morphology of the primary lesions and metastatic lesions of breast invasive ductal carcinoma with neuroendocrine differentiation were basically the same, and they were all non-lumen invasive ductal carcinoma of the breast. The antibody markers of this case of breast invasive ductal carcinoma were as follows: the primary and metastatic foci were all positive for E-cad, p120 and ER, while PR, HER2, GATA3, Mammaglobin and GCDFP-15 were all negative. The antibody markers of non-breast tissues were as follows: TTF1, CgA and Sym were positively expressed in primary and metastatic foci. This tumour was extremely rare and had characteristic histomorphological changes.

Conclusion: The diagnosis of invasive breast carcinoma with neuroendocrine differentiation should be based on histology and cell morphology, $\mathrm{CgA}$ and SyN-positive expression, mitosis and cell proliferation index ki-67 positive cell comprehensive analysis, in order to improve understanding and early diagnosis and avoid missed diagnosis and misdiagnosed cases.

\section{Introduction}

Breast cancer is the most common malignant tumour in women and the second leading cause of cancer mortality worldwide. Most deaths from breast cancer are caused by metastasis, which usually occurs in the bone, liver, lung, intramedullary, etc. Gastrointestinal metastasis of breast cancer is rare [1-3]. The metastasis rate of breast invasive lobular carcinoma (ILC) and invasive ductal carcinoma (IDC) is very different. Breast IDC metastases to the liver, lung and brain are more common. Breast IDC metastases to the gastrointestinal tract are extremely rare [4]. Breast IDC with neuroendocrine differentiation is a rare subtype of breast cancer. Distinguishing between primary and metastatic in histomorphology is challenging, especially when it is necessary to make a correct pathological diagnosis for some abnormal immunophenotypes. [5-7]. In this case report, the rareinvasive breast cancer with neuroendocrine differentiation that has metastases to the stomach, colon and lungs at the same time can help clinical diagnosis and differential diagnosis and avoid missed diagnosis and misdiagnosis.

\section{Materials And Methods}

1. Clinical data: 
The clinicopathological data of one patient with breast IDC and with simultaneous gastric, colon and lung metastases in the Department of Pathology, Shenzhen Hospital of Southern Medical University, China were collected. This patient was female, 51 years old, and came to our hospital for medical treatment on August 26, 2020.

\section{Methods:}

Biopsy specimens were fixed immediately with freshly prepared $10 \%$ neutral buffered formalin (NBF) solution for eight hrs, routinely dehydrated, embedded in paraffin and prepared into $4 \mu \mathrm{m}$ thick sections. The tissue structure and cell morphology were observed under a light microscope after HE staining.

\section{Immunohistochemical staining:}

The tissue sections were deparaffinised, hydrated and rinsed with distilled water using streptavidinperoxidase (SP) method. Then the slices were placed in TBS for ten minutes. Endogenous peroxidase was blocked for another five mins, and then the sections were treated with TBS for ten mins. Each primary antibody (CKpan, CK7, CK20, ER, PR, HER2, p120, E-cadherin, CATA3, Mammaglobin, GCDFP-15, TTF1, CDX2, Villin, ki-67) were incubated with the slices at room temperature for 30 mins. After washing for ten mins in TBS, the sections were incubated in EnVisionTM. After washing in TBS for another ten mins, the secondary antibody was applied for ten mins. The chromogenic substrate solution was incubated for ten minutes and then rinsed with distilled water. DAB was used to develop colour and counterstained with haematoxylin. A known gastric mucosal section was used as a positive control, and PBS buffer instead of the primary antibody was used as a negative control. The working solutions were purchased from Fuzhou Maixin Biotechnology Development Co., Ltd., and the operation steps were carried out in strict accordance with the kit instructions.

\section{Results}

\section{Clinical examination:}

The breast colour ultrasound in our hospital showed 'solid mass of right breast at 9 o'clock, BI-RADS classification: $₫ a$. There were multiple solid nodules in both breasts, with BI-RADS classification: Class III'. CT showed that 'the upper lobe of the left lung occupies, considering the possibility of lung cancer, more lymph nodes in the mediastinum, considering metastasis; the upper lobe of both lungs and the dorsal segment of the lower lobe were mostly scattered in nodules'. The right breast mass and lung mass were punctured, gastroscope and colonoscopy were biopsied, and all were sent for pathological examination.

\section{Histopathological characteristics and immunophenotype}




\subsection{Breast IDC}

The tumour tissue under the microscope showed solid nest-like, cluster-like, trabecular or adenoid structures, with very few fibrous tissue intervals, very little inflammatory cell infiltration and large necrotic foci (Figure 1A). The cytoplasm was rich and eosinophilic, and the nucleus was irregularly round or oval, accompanied by one or more small nucleoli. About $7 \%$ to $13 \%$ of the nuclei had larger eosinophilic nucleoli (Figure 1B). The mitotic figures were 4-9/10 high power fields (HPFs) with invasive tumour growth. E-cad (Figure 1C) and p120 (Figure 1D) were positively expressed on the cell membrane. CKpan and CK7 were positively expressed in the cytoplasm. ER (Figure 1E) was $+30 \%$. CgA (Figure 1F) was positively expressed in the cytoplasm, with the number of positive cells $>90 \%$. SyN was positively expressed with the number of positive cells $>50 \%$. Thyroid transcription factor 1 (TTF-1) (Figure 1G) was positively expressed in the nucleus. HER2, PR, GATA3, Mammaglobin, GCDFP-15, NapsinA, CDX2 and Villin were negatively expressed. ki67 (Figure $1 \mathrm{H})$ was $+20 \%$.

\subsection{Histological characteristics of gastric mucosal metastasis}

Under the microscope, the tumour was confined to the lamina propria of the gastric mucosa and was nodular in an area of $1.3 \mathrm{~cm} \times 0.8 \mathrm{~cm}$. There was erosion on the luminal margin of the tumour area. The tumour tissue formed a solid nest-like, cluster-like, trabecular or adenoid structure, with very few fibrous tissue intervals and very little inflammatory cell infiltration (Figure 2A). Tumour cells had different shapes, rich cytoplasm, eosinophilic, and irregular round or oval nuclei, accompanied by one or more nucleoli. The mitotic figures were 4-9/10 HPFs. The tumour tissue grew invasively, infiltrating the lamina propria of the gastric mucosa, with a single cell or multiple cells infiltrating the surrounding normal lamina propria, where there was a small amount of fibrous connective tissue and inflammatory cells. E-cad (Figure 2B) and p120 were positively expressed on the cell membrane, with ER+30\% (Figure 2C). CgA (Figure 2D) was positively expressed in the cytoplasmic, and SyN was positively expressed focally in the cytoplasmic. TTF1 was positively expressed (Figure 2E). HER2, PR, GATA3, Mammaglobin, GCDFP-15, NapsinA, CDX2 and Villin were all negatively expressed. ki67 was $+20 \%$.

\subsection{Histological characteristics of colonic liver flexure metastasis}

Under the microscope, the tumour was located in the colonic mucosa and submucosa, with an area of 2.2 $\mathrm{cm} \times 1.5 \mathrm{~cm}$, and the tumour tissue was infiltrating. The tumour tissue formed a solid nest-like, cluster-like, trabecular or adenoid structure, with very few fibrous tissue intervals and very little inflammatory cell infiltration (Figure 3A). The tumour cells had different shapes, rich cytoplasm, eosinophilic, and irregular round or oval nuclei, accompanied by one or more nucleoli. E-cad (Figure 3B) and p120 were positively expressed on the cell membrane, andER (Figure 3C) was +30\%. CgA (Figure 3D) was positively 
expressed in the cytoplasm, and SyN was positively expressed in the focal cytoplasm. TTF1 was positive (Figure 3E). HER2, PR, GATA3, Mammaglobin, GCDFP-15, NapsinA, CDX2 and Villin were all negatively expressed. ki67 was $+20 \%$.

\subsection{Histological characteristics of lung metastases}

Under the microscope, the tumour tissue formed solid nests, clusters, trabecular or adenoid structures, large coagulation necrosis and haemorrhage (Figure 4A). The tumour cells had different shapes, rich cytoplasm, eosinophilic, and irregular round or oval nuclei, accompanied by one or more nucleoli. The mitotic figures were 4-9/10 HPFs. The tumour tissue grew invasively, infiltrating the lamina propria of the gastric mucosa, with a single cell or multiple cells infiltrating the surrounding normal lamina propria, where there was a small amount of fibrous connective tissue and inflammatory cells. E-cad (Figure 4B) and $\mathrm{p} 120$ were positively expressed on the cell membrane, with ER $+30 \%$ (Figure 4C). CgA (Figure 4D) was positively expressed in the cytoplasmic, and SyN was positively expressed focally in the cytoplasmic. TTF1 was positive (Figure 4E). HER2, PR, GATA3, Mammaglobin, GCDFP-15, NapsinA, CDX2 and Villin were all negatively expressed. ki67 was $+20 \%$.

\section{Follow-up}

The patient came to our hospital for treatment from August 26,2020 , to June 26,2021 , and was followed up for a total of ten months. There was a telephone follow-up on February 17, 2021. The family members of the patient complained that the patient was taking $600 \mathrm{mg}$ of alectinib, which was acceptable. The dose was gradually increased to $900 \mathrm{mg}$, then $1200 \mathrm{mg}$. Tamoxifen endocrine therapy was continued. There was a telephone follow-up on June 14,2021 . The family members of the patient complained that the patient was in poor condition and came to the Department of Oncology of our hospital for hospitalisation the next day. The ward round record on June 26, 2021 showed that the patient was in a poor mental state, spoke slowly and complained of dizziness and discomfort. After eating, the patient had intermittent nausea and vomiting, poor appetite, obvious fatigue, good stools and not much urination. Blood routine: white blood cell count (WBC): $2.97 \times 10^{9} / \mathrm{L}$, absolute neutrophil count (NEUT\#): $1.92 \times 10^{9} / \mathrm{L}$, red blood cell count (RBC): $3.66 \times 10^{12} / \mathrm{L}$, haemoglobin (HGB): $125 \mathrm{~g} / \mathrm{L}$, platelet count (PLT): $269 \times 10^{9} / \mathrm{L}$. Liver function: alanine aminotransferase (ALT): $53.00 \mathrm{U} / \mathrm{L}$, transaminase ratio (AST/ALT): 0.58, total protein (TP): $61.00 \mathrm{~g} / \mathrm{L}$, albumin (Alb): $31.50 \mathrm{~g} / \mathrm{L}$, white/ball ratio (A/G): 1.07. Seven electrolytes: potassium (K): $3.14 \mathrm{mmol} / \mathrm{L}$, sodium (Na): $134.60 \mathrm{mmol} / \mathrm{L}$, chlorine $(\mathrm{Cl})$ 97.20: $\mathrm{mmol} / \mathrm{L}$, calcium (Ca): $2.05 \mathrm{mmol} / \mathrm{L}$, total carbon dioxide $\left(\mathrm{CO}_{2}\right): 30.40 \mathrm{mmol} / \mathrm{L}$; Fibrinogen (FIB): $1.81 \mathrm{~g} / \mathrm{L}$, D-Dimer (D-Dimer): $2.09 \mu \mathrm{g} / \mathrm{mL}$; carcinoembryonic antigen (CEA): $4800.00 \mathrm{ng} / \mathrm{mL}$, carbohydrate antigen (CA153): $402.00 \mathrm{U} / \mathrm{mL}$. Opinion of the attending physician on ward rounds: the patient had stage IV breast cancer on the right side with targeted therapy for lung and gastrointestinal mucosal metastasis and was currently experiencing severe malnutrition with weight loss. After the patient's symptoms had stabilised, imaging examination would be considered. Based on the patient's condition, the Nutrition Department 
recommended the following nutritional support programmes: $₫$ The target calorie for the whole day was $1855 \mathrm{kcal} / \mathrm{d}$; $\otimes T$ Three meals were given as a liquid diet; $\otimes T$ The patient was not eating enough by mouth, and it was recommended to assist intravenous nutrition in improving the nutritional status to prevent refeeding syndrome.

\section{Discussion}

So far, scientists have not found the exact cause of breast cancer. Fewer than $10 \%$ of breast cancers can be attributed to genetic mutations. Breast cancer is usually related to the environment, reproduction and lifestyle [8]. It has been reported in the literature that $75 \%$ of patients who underwent endoscopy or gastrointestinal surgery and were finally diagnosed with metastatic breast cancer wereprimary ILC. About $82 \%$ of patients had positive hormone receptors in metastatic lesions [9]. This case report was consistent with the literature report. When breast lobular carcinoma has gastric and intestinal metastasis, gastric metastasis of breast lobular cancer could be the first symptom. The patient was treated with neoadjuvant chemoradiation and total gastrectomy, and 14 months later, primary breast lobular carcinoma was discovered [8]. The reported case of breast IDC with gastric, colon and lung metastases at the same time was a very rare case. For those with gastrointestinal metastases that were the first onset and lack of a history of primary breast cancer, it was easy to misdiagnose [10].

For adenocarcinoma of the breast, lung, stomach and intestine, it is necessary to know how to determine the primary and metastatic lesions. Our experience is a comprehensive analysis of the histological structure and antibody markers in each part. When the primary breast cancer metastasises, the histopathological characteristics and immunophenotypes of the primary tumour and the metastasis are consistent with literature reports, but there are also literature reports that are inconsistent [11-13]. The histological structure and cell morphology of the primary breast cancer lesion and the metastatic lesions of lung, stomach and colon were basically the same, and there was no poorly differentiated invasive breast ductal carcinoma of the lumen. The results of breast IDC marker antibodies showed that the primary and metastatic lesions showed positive expressions of E-cad, p120 and ER and negative expressions of PR, HER2, GATA3, Mammaglobin and GCDFP-15. The results of non-breast tissue marker antibodies showed that TTF-1, CgA and SyN were positively expressed in the primary and metastatic foci. The above results suggested that the histologically non-luminal IDC of the breast and the immunophenotype of TTF-1, CgA and SyN antibody-positive expression were prone to multiple metastases throughout the body.

The tissue structure, cell morphology and immunophenotype of this case all pointed to IDC of the breast. Subsequent immunohistochemical staining showed that the number of $\mathrm{CgA}$-positive cells was $\geq 90 \%$; the number of SyN-positive cells was $\geq 50 \%$, which belonged to invasive breast cancer with neuroendocrine differentiation. On the one hand, the disease was easy to miss, and it was mainly not labelled with chromogranin A or synaptophysin, or both. On the other hand, it was easy to misdiagnose, mainly because only $\mathrm{CgA}$ and SyN were labelled to confirm neuroendocrine tumours. This report proposed the following key points of pathological diagnosis: $\triangle$ Those with histopathological features of IDC of the 
breast should be tested for both breast tissue-marked antibodies and non-breast tissue-marked antibodies; खlf it was confirmed histologically that the adenocarcinoma was strongly positive for CKpan, regardless of whether chromogranin A or synaptophysin was extensively or partially positive, it should be diagnosed as adenocarcinoma with neuroendocrine characteristics.

TTF-1 is usually used for immunohistochemical evaluation of lung cancer because TTF- 1 is positive in a large proportion of lung cancers and is usually negative in other adenocarcinomas [14]. In the case of ERpositive breast cancer, some areas of the primary tumour were also positive for TTF-1, but they completely lost ER expression at the site of metastasis and recurrence while retaining TTF-1 positive [15]. In this case, both the primary tumour and metastasis were positive for TTF- $1 \geq 90 \%$ and positive for ER $\geq 50 \%$. It is known that a small number of cases of breast cancer express TTF- 1 , but when this staining is used for the differential diagnosis of lung cancer, this may cause problems. In this study, the case with breast IDC metastasis to the stomach, colon and lung at the same time had positive expressions of TTF1. In this case, both the primary tumour and the metastasis showed strong positive expressions of TTF-1, and the breast cancer had metastasis to the gastric mucosa, which had metastasised to the gastric mucosa after one year, accompanied by lung and colon metastasis. IDC of breast cancer homologously metastasised to the stomach, colon and lung for more than one year, suggesting that the lesions developed rapidly and the metastasis rate was high. TTF-1 is considered to be a reliable marker of lung or thyroid origin, and it is only occasionally positively expressed in cases of primary breast cancer [15]. The differential diagnosis between primary and secondary breast cancer is difficult, especially in poorly differentiated tumours. TTF- 1 is positively expressed in primary breast cancer, indicating a poor prognosis and prone to metastasis.

In summary, it is very rare that invasive breast cancer with neuroendocrine differentiation has gastric, colon and lung metastases at the same time. This report suggests: खHow to determine the primary and metastatic lesions in the four parts of the breast, lung, stomach and intestine, mainly combined with histological morphology and immunophenotype comprehensive analysis; $\bigotimes$ The diagnosis of invasive breast cancer with neuroendocrine differentiation needs to be based on tissues structure and cell morphology, CgA and SyN-positive expression, mitoses and cell proliferation index ki-67 positive cells; \ln patients with breast IDC, those with positive immunophenotypes of TTF-1, CgA and Sym antibodies had a poor prognosis and were prone to multiple metastases throughout the body. The molecular biology and genetics of invasive breast cancer with neuroendocrine differentiation need to be accumulated and further studied in more cases.

\section{Abbreviations}

ILC: invasive lobular carcinoma

IDC: invasive ductal carcinoma

NBF: neutral buffered formalin 


\section{Declarations}

\section{Ethics approval and consent to participate}

This study was conducted in accordance with the Declaration of Helsinki and approved by the ethics committee of Shenzhen Hospital, Southern Medical University.The patient agreed to participate and signed the informed consent form.

\section{Competing interests}

All of the authors had no any personal, financial, commercial, or academic conflicts of interest separately.

\section{Consent for publication}

Not applicable.

\section{Availability of data and materials}

All data generated or analyzed during this study are included in this published article

\section{Funding}

Fund Project: Key Science and Technology Research Plan Project in Henan Province (132102310008)

\section{Authors' contributions}

Wang YK conceived of the study, and Liao XH , Yang BF participated in its design and coordination and Chen XD helped to draft the manuscript. All authors read and approved the final manuscript.

\section{Acknowledgements}

Not applicable. 
1. Haider MT, Smit DJ, Taipaleenmäki H. The Endosteal Niche in Breast Cancer Bone Metastasis. Front Oncol. 2020 Mar 13; 10:335.

2. Bale R, Putzer D, Schullian P. Local Treatment of Breast Cancer Liver Metastasis. Cancers (Basel). 2019 Sep 11;11(9):1341.

3. Rostami R, Safarpour D, Tavassoli FA, Jabbari B. Intramedullary metastasis in breast cancer-a comprehensive literature review. J Neurol Sci. 2013 Sep 15; 332(1-2):16-20.

4. de Zárraga Mata C, Thomas Salom G, Vilella Martorell A, Salvà Ramonell F, Maura Oliver ÁL, Dolz Abadía C. Gastric metastatic extension of invasive ductal carcinoma of the breast with atypical endoscopic presentation. Gastroenterol Hepatol. 2018 May; 41(5):304-305.

5. Birla R, Dinu D, losif C, Constantinoiu S. Gastric Metastasis of Invasive Lobular Breast Carcinoma, a Current Diagnostic and Treatment Challenge - A Review. Chirurgia (Bucur). 2019 Sept-Oct; 114(5):571-578.

6. Critchley AC, Harvey J, Carr M, Iwuchukwu O. Synchronous gastric and colonic metastases of invasive lobular breast carcinoma: case report and review of the literature. Ann R Coll Surg Engl. 2011 Jul; 93(5):e49-50.

7. Aurello P, D'Angelo F, Cosenza G, Petrocca S, Stoppacciaro A, Ramacciato G, Ziparo V. Gastric metastasis 14 years after mastectomy for breast lobular carcinoma: case report and literature review. Am Surg. 2006 May; 72(5):456-460.

8. Dos Santos Fernandes G, Batista Bugiato Faria LD, de Assis Pereira I, Neves NC, Vieira YO, Leal Al. Gastric Metastasis of Breast Cancer: A Case Series. Rare Tumors. 2016 Oct 6; 8(3):6305.

9. Ongaro E, Gerratana L, Cinausero M, Pelizzari G, Poletto E, Giangreco M, Andreetta C, Pizzolitto S, Di Loreto C, Minisini AM, Mansutti M, Russo S, Fasola G, Puglisi F. Comparison of primary breast cancer and paired metastases: biomarkers discordance influence on outcome and therapy. Future Oncol. 2018 Apr; 14(9):849-859.

10. Buka D, Dvořák J, Richter I, Hadzi ND, Cyrany J. Gastric and Colorectal Metastases of Lobular Breast Carcinoma: A Case Report. Acta Medica (Hradec Kralove). 2016; 59(1):18-21.

11. Shi YJ, Tsang JY, Ni YB, Tse GM. Intratumoral Heterogeneity in Breast Cancer: A Comparison of Primary and Metastatic Breast Cancers. Oncologist. 2017 Apr; 22(4):487-490.

12. Kulka J, Székely B, Lukács LV, Kiss O, Tőkés AM, Vincze E, Turányi E, Fillinger J, Hanzély Z, Arató G, Szendrői M, Győrffy B, Szász AM. Comparison of Predictive Immunohistochemical Marker Expression of Primary Breast Cancer and Paired Distant Metastasis using Surgical Material: A Practice-Based Study. J Histochem Cytochem. 2016 Apr; 64(4):256-267.

13. Matsutani S, Tanaka H, Sakurai K, Lee T, Toyokawa T, Kubo N, Muguruma K, Kimura K, Nagahara H, Noda E, Amano R, Maeda K, Sawada T, Ohira M, Hirakawa K. A case of metastatic gastric cancer from breast cancer treated with proximal gastrectomy. Gan To Kagaku Ryoho. 2013 Nov; 40(12):2210-2213.

14. Voutsadakis IA, Mozarowski P. Expression of TTF-1 in breast cancer independently of ER expression: A case report and pathogenic implications. Breast Dis. 2017; 37(1):1-6. 
15. Klingen TA, Chen Y, Suhrke P, Stefansson IM, Gundersen MD, Akslen LA. Expression of thyroid transcription factor- 1 is associated with a basal-like phenotype in breast carcinomas. Diagn Pathol. 2013 May 15; 8:80.

\section{Figures}
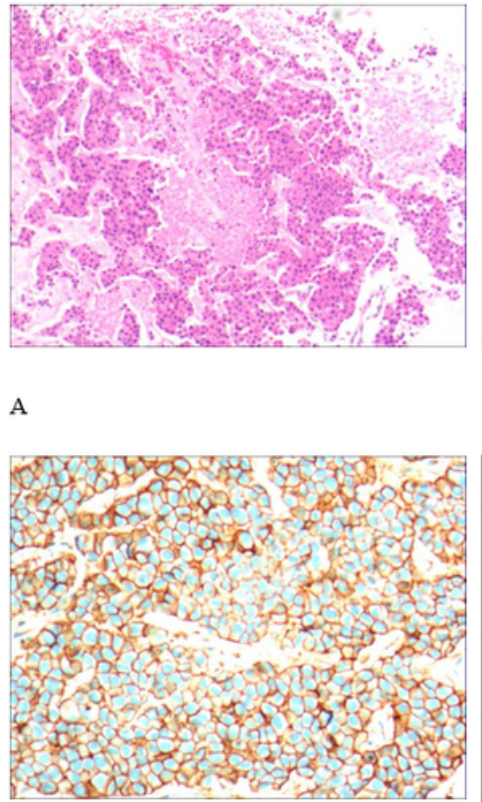

$\mathrm{c}$

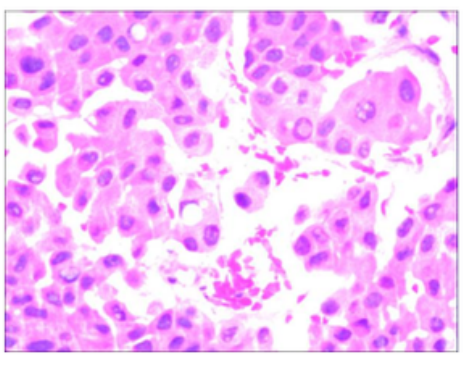

B

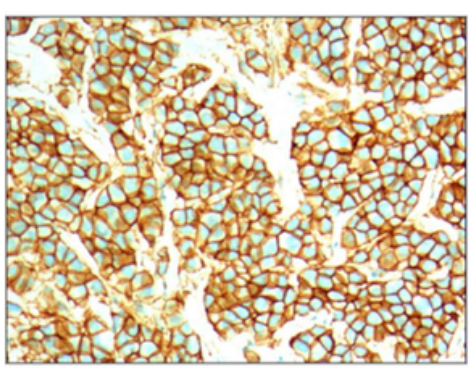

D

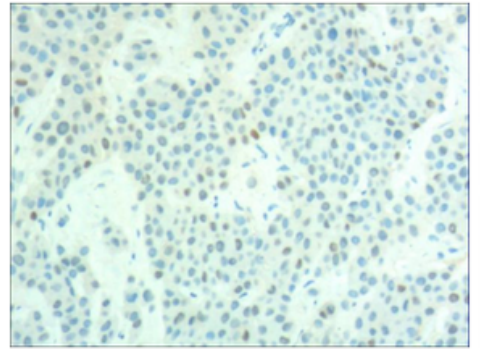

E

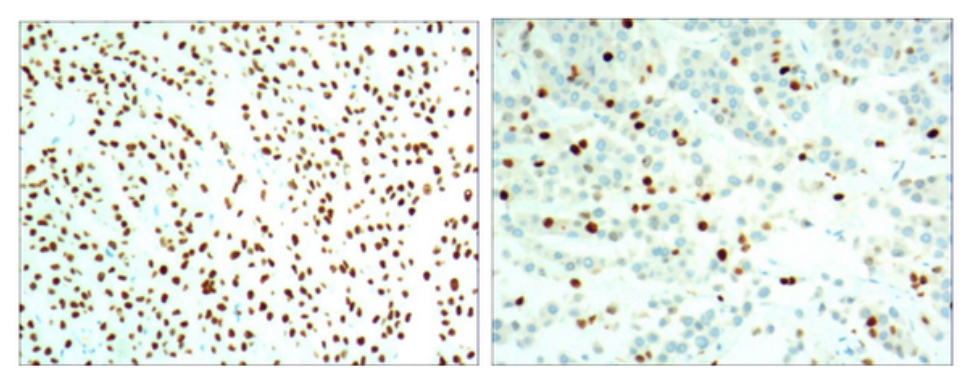

G

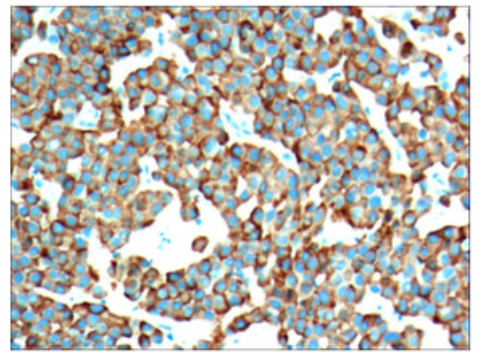

F

H

\section{Figure 1}

Invasive ductal carcinoma of breast cancer

The tumour tissue formed a solid nest-like, cluster-like, trabecular or adenoid structure, with few fibrous tissue intervals and large areas of necrosis, H\&E×40. B. Cytologically, rich cytoplasm, eosinophilic. The nucleus was irregularly round or oval, with one or more nucleoli, H\&E×200. C. E-cad was positively expressed on the cell membrane, SP method $\times 100$. D. p120 was positively expressed on the cell membrane, SP method $\times 100$. E. ER hot spot area $+30 \%$, SP method $\times 100$. F. CgA was positively expressed in the nucleus, SP method $\times 100$. G. TTF1 was positively expressed in the nucleus, SP method $\times 100$. H. ki67 Hot spot area $+20 \%$, SP method $\times 100$.

\section{Figure 2}

Gastric mucosal metastasis

The tumour was confined to the lamina propria of the gastric mucosa and was nodular, with an area of $1.3 \mathrm{~mm} \times 0.8 \mathrm{~mm}$. There was erosion on the luminal margin of the tumour area, and the tumour tissue 
formed solid nests, clusters, trabeculae or adenoid structures, with very few fibrous tissue compartments, very few inflammatory cell infiltration, $\mathrm{H} \& \mathrm{E} \times 40$. B. E-cad was positively expressed on the cell membrane, SP method $\times 100$. C. ER hot spot area $+30 \%$, SP method $\times 100$. D. CgA was positively expressed in the nucleus, SP method $\times 100$. E. TTF1 was positively expressed, SP method $\times 100$.

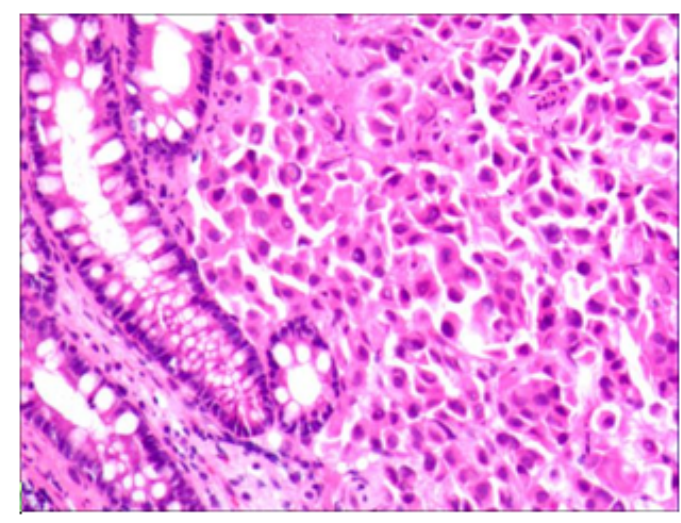

A

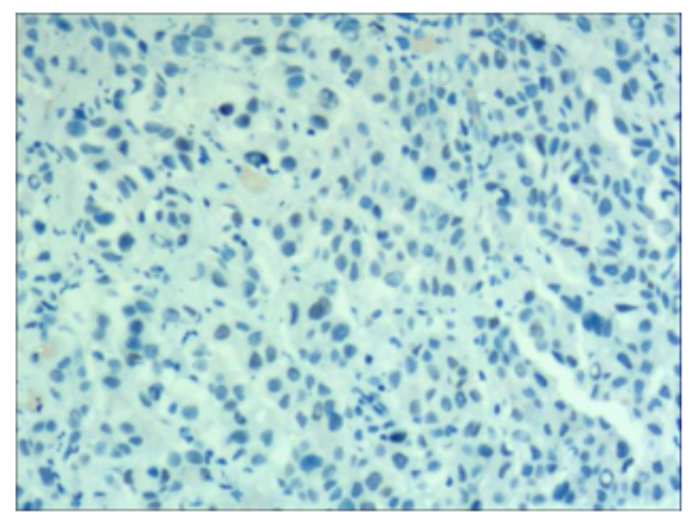

$\mathrm{C}$

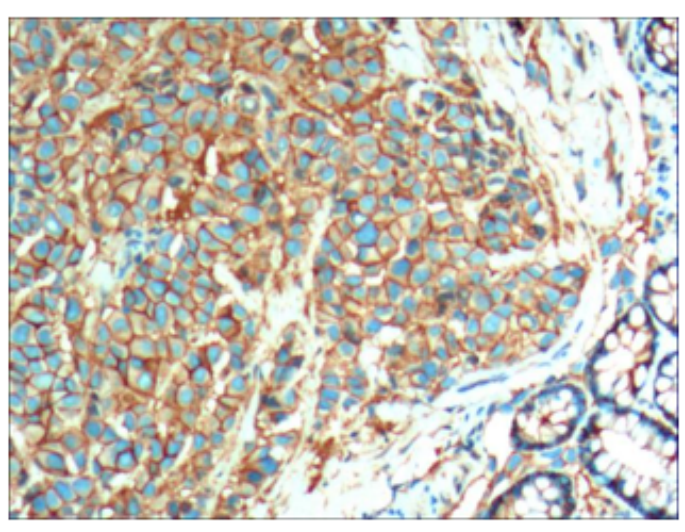

B

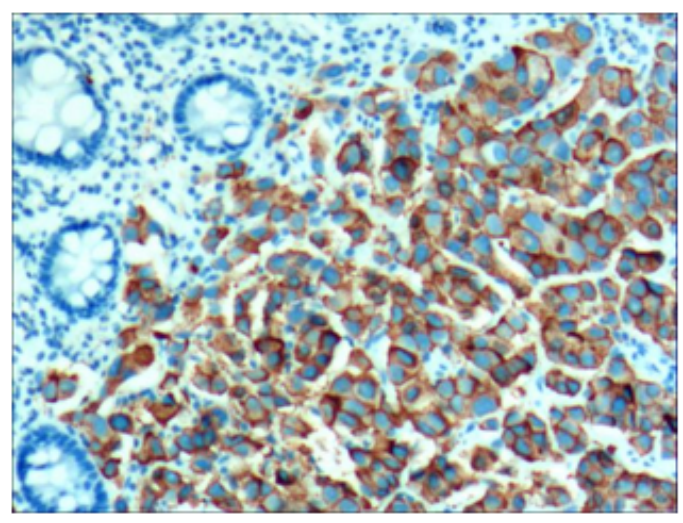

$\mathrm{D}$

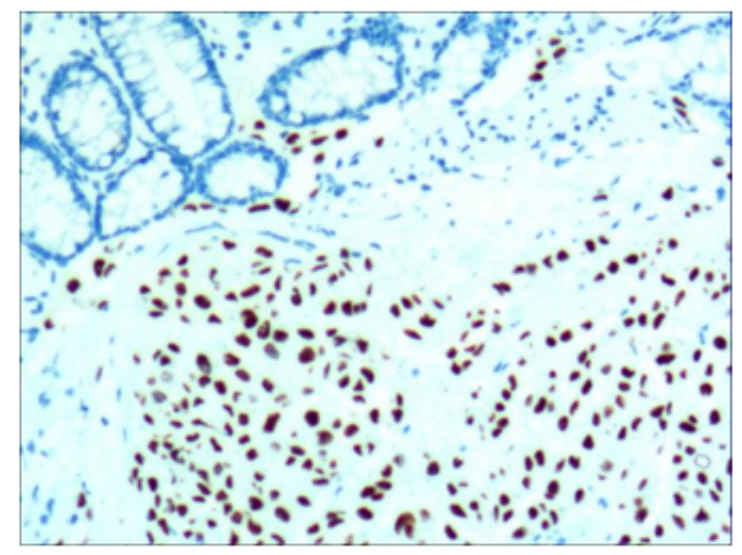

E

\section{Figure 3}


The tumour was located in the colonic mucosa and submucosa. It was nodular, with an area of $1.3 \mathrm{~mm}$ $\times 0.8 \mathrm{~mm}$. The tumour tissue formed a solid nest-like, cluster-like, trabecular or adenoid structure with very few fibres, very few inflammatory cell infiltration, $H \& E \times 100$. B. E-cad was positively expressed on the cell membrane, SP method $\times 100$. C. ER hot spot area $+30 \%$, SP method $\times 100$. D. CgA was positively expressed in the nucleus, SP method $\times 100$. E. TTF1 was positively expressed, SP method $\times 100$.
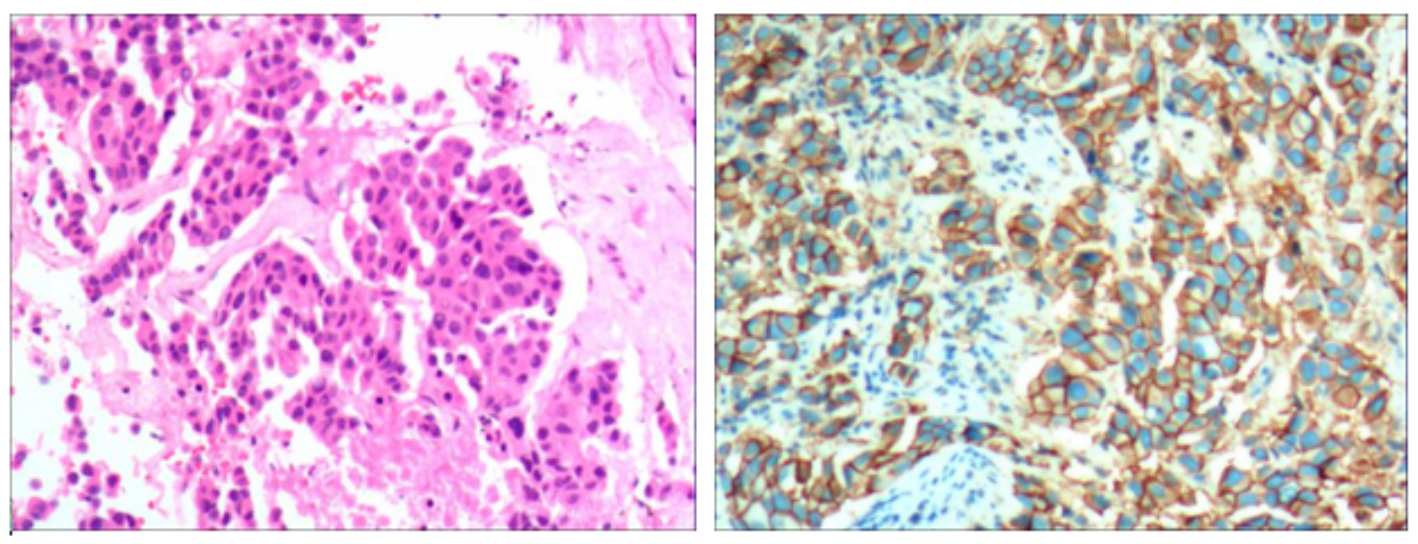

A

B
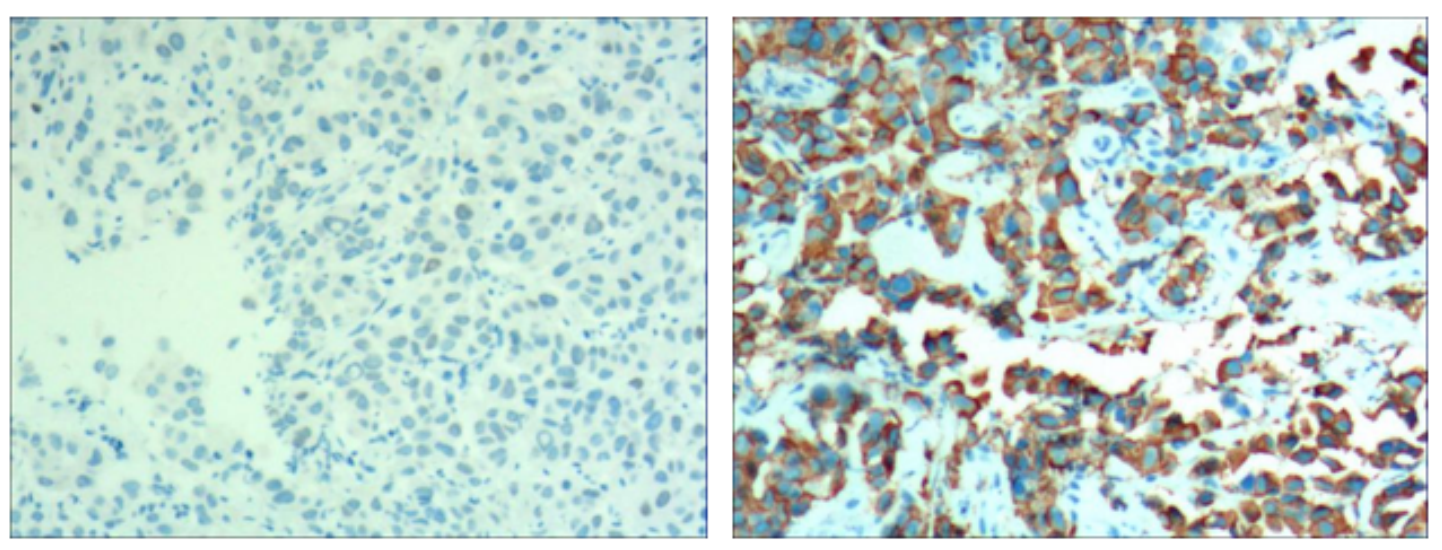

$\mathrm{C}$

$\mathrm{D}$

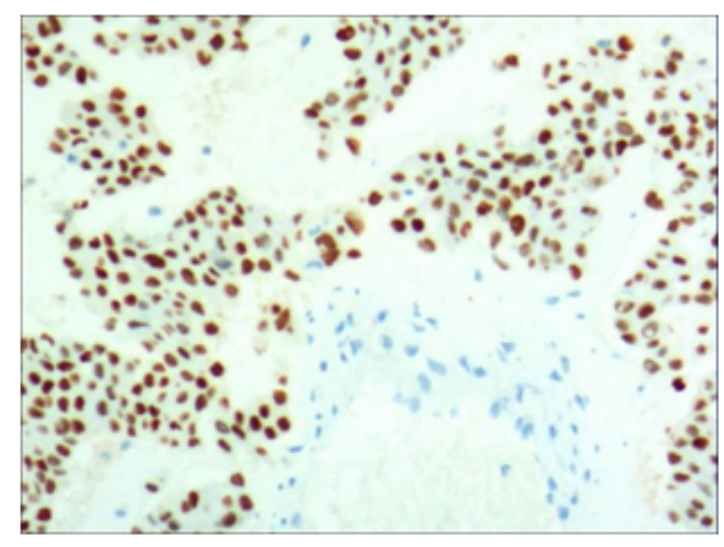

E 


\section{Figure 4}

\section{Lung metastasis}

The tumour was confined to the lamina propria of the gastric mucosa and was nodular, with an area of $1.3 \mathrm{~mm} \times 0.8 \mathrm{~mm}$. There was erosion on the luminal margin of the tumour area, and the tumour tissue formed solid nests, clusters and trabeculae or adenoid structures, with very few fibrous tissue compartments, very few inflammatory cell infiltration, H\&E×40. B. E-cad was positively expressed on the cell membrane, SP method $\times 100$. C. ER hot spot area $+30 \%$, SP method×100. D. CgA was positively expressed in the nucleus, SP method×100. E. TTF1 was positively expressed, SP method×100. 